\title{
MEROMORPHIC FUNCTIONS SHARING THREE VALUES OR SETS CM
}

\author{
PING LI
}

\begin{abstract}
In this paper, by using a lemma about common 1-points, the author studied the relationship between two meromorphic functions which share three values or three sets $\mathrm{CM}$, and generalised a result obtained recently by E. Mues [4], and weaked the condition of a theorem in [10].
\end{abstract}

\section{Introduction}

Let $f$ and $g$ be two non-constant meromorphic functions in the complex plane $C$ and $a$ be a value in the extended complex plane $\bar{C}$. We say that $f$ and $g$ share the value $a \mathrm{CM}(\mathrm{IM})$ provided that $f-a$ and $g-a(1 / f$ and $1 / g$, resp.) have the same zeros counting multiplicities (ignoring multiplicities) in the case of $a \in C(a=\infty$, resp.). For a positive integer or infinity $k$ let

$$
E(a, k, f)=\{z: f(z) \stackrel{(p)}{=} a, p \leq k\}
$$

here $p$ is the multiplicity of $a$-point of $f$. Then $f$ and $g$ share the value $a$ IM can be expressed as $E(a, \infty, f)=E(a, \infty, g) . \quad E(a, 1, f)=E(a, 1, g)$ means that $f$ and $g$ have the same simple $a$-points. Let

$$
E_{f}\{a\}=\{z \mid f(z)=a ; \text { counting multiplicities }\}
$$

and

$$
\tilde{E}_{f}\{a\}=\{z \mid f(z)=a \text {; ignoring multiplicities }\} .
$$

Then $f$ and $g$ share the value $a \mathrm{CM}$ (IM) can be expressed as $E_{f}\{a\}=E_{g}\{a\}$ $\left(\tilde{E}_{f}\{a\}=\tilde{E}_{g}\{a\}\right)$. A natural generalization of the concept "sharing a value" will be "sharing a set". The following two notations are natural and obvious.

$$
E_{f}(S)=\bigcup_{a \in S} E_{f}\{a\}, \quad \tilde{E}_{f}(S)=\bigcup_{a \in S} \tilde{E}_{f}\{a\},
$$

1991 Mathematics Subject Classification. Primary 30D35.

Key words and phrases. meromorphic function, entire function, sharing value, sharing set. Received September 16, 1997; revised April 16, 1998. 
where $S$ denotes an arbitrary set in $\overline{\boldsymbol{C}}$. For two meromorphic functions $f, g$ and a subset $S$ in $\overline{\boldsymbol{C}}$, we say that $f$ shares $S$ CM (IM) with $g$ provided that

$$
E_{f}(S)=E_{g}(S) \quad\left(\tilde{E}_{f}(S)=\tilde{E}_{g}(S)\right) .
$$

It is well known [5] that if two non-constant meromorphic functions $f$ and $g$ share four values CM, then $f$ is a Möbius transformation of $g$. In the case that $f$ and $g$ share three values CM, $f$ may not be a Möbius transformation of $g$, however, $f$ can still be, if some other appropriate condition is added. There have been published many papers to talk about meromorphic functions sharing three values CM (see, [2], [1], [11], [9] and [7]). In 1983, Ueda [8] proved that if $f$ and $g$ share $0,1, \infty \mathrm{CM}$ and if there exists an $a \neq 0,1, \infty$ such that $E(a, k, f)=$ $E(a, k, g)$ for some $k \geq 2$, then $f$ is a Möbius transformation of $g$. The bound 2 for $k$ here is best possible. For examples, functions

$$
f=\frac{e^{3 \gamma}-1}{e^{\gamma}-1} \quad \text { and } \quad g=\frac{e^{-3 \gamma}-1}{e^{-\gamma}-1}
$$

share $0,1, \infty \mathrm{CM}$ and satisfy $E(3 / 4,1, f)=E(3 / 4,1, g)$. Functions

$$
f=\frac{e^{\gamma}-1}{-e^{2 \gamma}-1} \quad \text { and } \quad g=\frac{e^{-\gamma}-1}{-e^{-2 \gamma}-1}
$$

share $0,1, \infty \mathrm{CM}$ and satisfy $E(a, 1, f)=E(a, 1, g)$, where $a$ is one of the solution of

$$
\frac{1}{4 a^{2}}=1-\frac{1}{a}
$$

Recently, E. Mues [4] proved the following theorem.

THEOREM A. Let $f$ and $g$ be non-constant meromorphic functions sharing $0,1, \infty C M$. Suppose additionally that there exists an $a \neq 0,1, \infty$ such that $E(a, 1, f)=E(a, 1, g)$. If $f$ is not a Möbius transformation of $g$, then there exists a Möbius transformation $L$ permuting $\{0,1, \infty\}$ such that $L \circ f$ and $L \circ g$ have the form (1) with $L(a)=3 / 4$ or $L \circ f$ and $L \circ g$ have the form (2) and $L(a)$ is a solution of (3).

For functions sharing sets, Gross-Yang [3] asked whether there exist two sets $S_{1}=\left\{a_{1}, a_{2}\right\}$ and $S_{2}=\left\{b_{1}, b_{2}\right\}$ such that for any two non-constant entire functions $f$ and $g$ the conditions $E_{f}\left(S_{j}\right)=E_{g}\left(S_{j}\right)(j=1,2)$ imply $f \equiv g$ or not. F. Gross and C. C. Yang studied the question for the case $a_{1}+a_{2}=b_{1}+b_{2}$, and H. X. Yi [10] improved Gross-Yang's result in the following manner.

THEOREM B. Let $f$ and $g$ be non-constant entire functions with finite order and $S_{1}=\left\{a_{1}, a_{2}\right\}, \quad S_{2}=\left\{b_{1}, b_{2}\right\}$ satisfying $a_{1} \neq a_{2}, \quad b_{1} \neq b_{2}, a_{1}+a_{2}=b_{1}+b_{2}=c$, $a_{1} a_{2} \neq b_{1} b_{2}$. If $E_{f}\left(S_{j}\right)=E_{g}\left(S_{j}\right)(j=1,2)$, then $f$ and $g$ assume one of the following relations: 
(i) $f \equiv g$,

(ii) $f+g \equiv c$,

(iii) $\left(f-\frac{c}{2}\right)\left(g-\frac{c}{2}\right) \equiv \pm\left(\frac{a_{1}-a_{2}}{2}\right)^{2}$, with $\left(a_{1}-a_{2}\right)^{2}+\left(b_{1}-b_{2}\right)^{2}=0$,

(iv) $\left(f-a_{j}\right)\left(g-a_{k}\right) \equiv(-1)^{j+k}\left(a_{1}-a_{2}\right)^{2}(j, k=1,2), \quad$ with $\quad 3\left(a_{1}-a_{2}\right)^{2}+$ $\left(b_{1}-b_{2}\right)^{2}=0$

(v) $\left(f-b_{j}\right)\left(g-b_{k}\right) \equiv(-1)^{j+k}\left(b_{1}-b_{2}\right)^{2}(j, k=1,2), \quad$ with $\quad\left(a_{1}-a_{2}\right)^{2}+$ $3\left(b_{1}-b_{2}\right)^{2}=0$.

In this paper, noting that the assumptions of Theorem $\mathrm{A}$ leads to $\bar{N}_{1)}(r, 1 /(f-a))=S(r, f)$, we will replace the condition $E(a, 1, f)=E(a, 1, g)$ by $\bar{N}_{1)}(r, 1 /(f-a))=S(r, f)$, and give a generalization of Theorem A. Also we will remove the limitation to the order in Theorem B.

We assume that the readers are familiar with the basic notations and results in value distribution theory. We use $S(r, f)$ to denote the quantity $o(T(r, f))$, $r \rightarrow \infty, r \notin E$, here and in the sequel, the letter $E$ is a set of $r \in(0, \infty)$ with finite linear measure not necessarily the same at each occurrence. $\bar{N}_{k)}(r, 1 /(f-a))$ denotes the reduced counting function of $a$-points of $f$ with multiplicities less than or equal to $k$. Similar notation $\bar{N}_{(k}(r, 1 /(f-a))$ is also used to denote the reduced counting function of $a$-points of $f$ with multiplicities more than or equal to $k$.

\section{Lemmas and results}

The following lemmas will be used in the proofs of our theorems.

LEMMA 1 (see [6]). Let $f_{1}$ and $f_{2}$ be two non-constant meromorphic functions satisfying

$$
\bar{N}\left(r, f_{\imath}\right)+\bar{N}\left(r, \frac{1}{f_{i}}\right)=S\left(r ; f_{1}, f_{2}\right), \quad i=1,2 .
$$

If $f_{1}^{s} f_{2}^{t}-1$ is not identically zero for arbitrary integers $s$ and $t(|s|+|t|>0)$, then for any positive number $\varepsilon$, we have

$$
N_{0}\left(r, 1 ; f_{1}, f_{2}\right) \leq \varepsilon T(r)+S\left(r ; f_{1}, f_{2}\right),
$$

where $N_{0}\left(r, 1 ; f_{1}, f_{2}\right)$ denotes the reduced counting function of $f_{1}$ and $f_{2}$ related to the common 1-points of $f_{1}$ and $f_{2}$, and $T(r)=T\left(r, f_{1}\right)+T\left(r, f_{2}\right), S\left(r ; f_{1}, f_{2}\right)=$ $o(T(r))$ as $r \rightarrow \infty, r \notin E$.

LEMMA 2 (see [6]). Let $f_{1}, f_{2}, \ldots, f_{n}$ be non-constant meromorphic functions satisfying

$$
\bar{N}\left(r, f_{l}\right)+\bar{N}\left(r, \frac{1}{f_{i}}\right)=S(r), \quad i=1,2, \ldots, n,
$$


and

$$
T\left(r, f_{i}\right) \neq S(r), \quad T\left(r, \frac{f_{i}}{f_{J}}\right) \neq S(r), \quad i \neq j, i, j=1,2, \ldots, n .
$$

Let $a_{0}, a_{1}, \ldots, a_{m}(m \leq n)$ be meromorphic functions satisfying $T\left(r, a_{i}\right)=S(r)$, $i=0,1, \ldots, m$. If

$$
\sum_{l=1}^{m} a_{i} f_{l} \equiv a_{0}
$$

then $a_{0} \equiv a_{1} \equiv \cdots \equiv a_{m} \equiv 0$, where $S(r)=o(T(r))$, as $r \rightarrow \infty$ and $r \notin E$, and $T(r)=\sum_{l=1}^{n} T\left(r, f_{i}\right)$.

LEMMA 3 (see [4]) and ([12]). Let $f$ and $g$ be meromorphic functions sharing $0,1, \infty C M$ and suppose that $f$ is not a Möbius transformation of $g$. Then we have

$$
T(r, f) \leq 2 \bar{N}\left(r, \frac{1}{f-a}\right)+S(r, f), \quad \text { for } a \neq 0,1, \infty .
$$

Now we state and prove the main theorems.

THEOREM 1. Let $f$ and $g$ be non-constant meromorphic functions sharing $0,1, \infty C M$. Suppose additionally that $f$ is not a Möbius transformation of $g$ and that there exists an $a \neq 0,1, \infty$ such that

$$
T(r, f) \leq c \bar{N}_{(2}\left(r, \frac{1}{f-a}\right)+S(r, f),
$$

here $c>0$ is a constant, then there exist a non-constant entire function $\gamma$, a nonzero constant $\lambda$ and two integers $s, t(t>0)$ which are mutually prime, such that

$$
\begin{gathered}
f=\frac{e^{t \gamma}-1}{\lambda e^{-s \gamma}-1}, \quad g=\frac{e^{-t \gamma}-1}{\frac{1}{\lambda} e^{s \gamma}-1}, \\
\frac{(1-a)^{s+t}}{a^{t}}=\lambda^{t} \frac{(1-\theta)^{s+t}}{\theta^{t}},
\end{gathered}
$$

with $\theta=-t / s \neq 1, a$.

Proof. Since $f$ and $g$ share 0,1 and $\infty \mathrm{CM}$, there exist two entire functions $\alpha$ and $\beta$ such that

$$
f=\frac{e^{\alpha}-1}{e^{\beta}-1}, \quad g=\frac{e^{-\alpha}-1}{e^{-\beta}-1} .
$$

Obviously, $\alpha, \beta$ and $\beta-\alpha$ are not constants, otherwise, $f$ is a Möbius transformation of $g$. By Nevanlinna second fundamental theorem, it is quite easy to get $T(r, f) \leq 3 T(r, g)+S(r, f)$ and $T(r, g) \leq 3 T(r, f)+S(r, g)$, and thus 
$S(r, f)=S(r, g)$. We use $S(r)$ to express $S(r, f)$ or $S(r, g)$. Formulas in (6) are equivalent to

$$
\frac{f}{g}=e^{\alpha-\beta}, \quad \frac{f-1}{g-1}=e^{\alpha},
$$

from which, we deduce that

$$
\begin{aligned}
T\left(r, e^{\alpha}\right) & \leq T(r, f)+T(r, g)+O(1) \\
& \leq 4 T(r, f)+S(r),
\end{aligned}
$$

and

$$
T\left(r, e^{\beta}\right) \leq 10 T(r, f)+S(r) .
$$

Hence we have $S\left(r, e^{\alpha}\right) \leq S(r)$ and $S\left(r, e^{\beta}\right) \leq S(r)$. On the other hand, from (6), we have $S(r) \leq S\left(r ; e^{\alpha}, e^{\beta}\right)$. Hence

$$
S(r)=S\left(r ; e^{\alpha}, e^{\beta}\right) .
$$

Let $z_{0}$ be a multiple $a$-point of $f$ but not a zero of $\alpha^{\prime}, \beta^{\prime}$ and $\beta^{\prime}-\alpha^{\prime}$. Then from

$$
f-a=\frac{e^{\alpha}-a e^{\beta}+a-1}{e^{\beta}-1}
$$

we see that

$$
\begin{gathered}
e^{\alpha\left(z_{0}\right)}-a e^{\beta\left(z_{0}\right)}+a-1=0, \\
\alpha^{\prime}\left(z_{0}\right) e^{\alpha\left(z_{0}\right)}-a \beta^{\prime}\left(z_{0}\right) e^{\beta\left(z_{0}\right)}=0,
\end{gathered}
$$

which lead to

$$
e^{\alpha\left(z_{0}\right)}=\frac{(1-\alpha) \beta^{\prime}\left(z_{0}\right)}{\beta^{\prime}\left(z_{0}\right)-\alpha^{\prime}\left(z_{0}\right)}, \quad e^{\beta\left(z_{0}\right)}=\frac{(1-a) \alpha^{\prime}\left(z_{0}\right)}{a\left(\beta^{\prime}\left(z_{0}\right)-\alpha^{\prime}\left(z_{0}\right)\right)} .
$$

Let

$$
F_{1}=\frac{\left(\beta^{\prime}-\alpha^{\prime}\right)}{(1-a) \beta^{\prime}} e^{\alpha}, \quad F_{2}=\frac{a\left(\beta^{\prime}-\alpha^{\prime}\right)}{(1-a) \alpha^{\prime}} e^{\beta} .
$$

Then from (10) and (15), we get

$$
\begin{aligned}
& T\left(r, F_{1}\right)=T\left(r, e^{\alpha}\right)+S\left(r ; e^{\alpha}, e^{\beta}\right), \\
& T\left(r, F_{2}\right)=T\left(r, e^{\beta}\right)+S\left(r ; e^{\alpha}, e^{\beta}\right),
\end{aligned}
$$

and thus

$$
S\left(r ; F_{1}, F_{2}\right)=S\left(r ; e^{\alpha}, e^{\beta}\right) .
$$


Since $F_{1}\left(z_{0}\right)=1, F_{2}\left(z_{0}\right)=1$, we have

$$
\bar{N}_{(2}\left(r, \frac{1}{f-a}\right) \leq N_{0}\left(r, 1 ; F_{1}, F_{2}\right)+S\left(r ; F_{1}, F_{2}\right) .
$$

From (16), (8), (9), (17) and the assumption, we get

$$
T\left(r, F_{1}\right)+T\left(r, F_{2}\right) \leq 14 c N_{0}\left(r, 1 ; F_{1}, F_{2}\right)+S\left(r ; F_{1}, F_{2}\right) .
$$

It is obvious that

$$
\bar{N}\left(r, F_{i}\right)+\bar{N}\left(r, \frac{1}{F_{i}}\right)=S\left(r ; F_{1}, F_{2}\right), \quad i=1,2 .
$$

Hence by Lemma 1, we see that there exist two non-zero and mutually prime integers $s, t(t>0)$ such that $F_{1}^{s} F_{2}^{t} \equiv 1$, i.e.,

$$
e^{s \alpha+t \beta} \equiv\left(\frac{(1-a) \beta^{\prime}}{\beta^{\prime}-\alpha^{\prime}}\right)^{s}\left(\frac{(1-a) \alpha^{\prime}}{a\left(\beta^{\prime}-\alpha^{\prime}\right)}\right)^{t}:=\rho,
$$

from which, we can see that $\rho^{\prime} / \rho=s \alpha^{\prime}+t \beta^{\prime}$. On the other hand, from the second equality in (19), we have

$$
\begin{aligned}
\frac{\rho^{\prime}}{\rho} & =t \frac{\left(\frac{\alpha^{\prime}}{\beta^{\prime}}\right)^{\prime}}{\left(\frac{\alpha^{\prime}}{\beta^{\prime}}\right)}-(s+t) \frac{-\left(\frac{\alpha^{\prime}}{\beta^{\prime}}\right)^{\prime}}{\left(1-\frac{\alpha^{\prime}}{\beta^{\prime}}\right)} \\
& =\frac{t+s \frac{\alpha^{\prime}}{\beta^{\prime}}}{\frac{\alpha^{\prime}}{\beta^{\prime}}\left(1-\frac{\alpha^{\prime}}{\beta^{\prime}}\right)}\left(\frac{\alpha^{\prime}}{\beta^{\prime}}\right)^{\prime} .
\end{aligned}
$$

If $\alpha^{\prime} / \beta^{\prime} \not \equiv-t / s$, then we have

$$
\alpha^{\prime} \equiv \frac{\left(\frac{\alpha^{\prime}}{\beta^{\prime}}\right)^{\prime}}{1-\frac{\alpha^{\prime}}{\beta^{\prime}}}
$$

and thus

$$
e^{\alpha}\left(1-\frac{\alpha^{\prime}}{\beta^{\prime}}\right) \equiv c_{1},
$$

where $c_{1}$ is a non-zero constant. Above identity can be rewritten as

$$
\beta^{\prime} \equiv \frac{\alpha^{\prime} e^{\alpha}}{e^{\alpha}-c_{1}}
$$


from which, we get

$$
e^{\beta} \equiv c_{2}\left(e^{\alpha}-c_{1}\right)
$$

where $c_{2}$ is also a non-zero constant. Formulas (20) and (21) imply $T\left(r, e^{\alpha}\right)=$ $S(r)$ and $T\left(r, e^{\beta}\right)=S(r)$. And thus from (6), we get $T(r, f)=S(r)$, a contradiction. Hence

$$
\frac{\alpha^{\prime}}{\beta^{\prime}} \equiv-\frac{t}{s}
$$

If $a=-t / s$, then $a \equiv \alpha^{\prime} / \beta^{\prime}$. Hence $e^{\alpha\left(z_{0}\right)}=1$ and $e^{\beta\left(z_{0}\right)}=1$. In the case that $z_{0}$ is a zero of $e^{\alpha}-a e^{\beta}+a-1$ with multiplicity not less than 3 , we have

$$
\alpha^{\prime \prime}\left(z_{0}\right)+\left(\alpha^{\prime}\left(z_{0}\right)\right)^{2}-a\left[\beta^{\prime \prime}\left(z_{0}\right)+\left(\beta^{\prime}\left(z_{0}\right)\right)^{2}\right]=0 .
$$

This and $a \equiv \alpha^{\prime} / \beta^{\prime}$ lead to $a(a-1) \beta^{\prime}\left(z_{0}\right)=0$, which contradicts the assumption about $z_{0}$. Hence $a \neq-t / s$.

Let $s \alpha+t \beta=c_{0}$, it is obviously a constant, and let $\gamma=\alpha / t$. Then $f$ and $g$ can be expressed as

$$
f=\frac{e^{t \gamma}-1}{\lambda e^{-s \gamma}-1}, \quad g=\frac{e^{-t \gamma}-1}{\frac{1}{\lambda} e^{s \gamma}-1},
$$

where $\lambda=e^{c_{0} / t}$. From (19) and (22), we have

$$
\frac{(1-a)^{s+t}}{a^{t}}=\lambda^{t} \frac{(1-\theta)^{s+t}}{\theta^{t}}
$$

where $\theta=-t / s \equiv \alpha^{\prime} / \beta^{\prime} \neq 1$, which completes the proof of Theorem 1 .

The following is an example which shows the existence of $f$ and $g$ in Theorem 1. and

Example 1. Let $\gamma$ be a non-constant entire function, $a=(20+4 \sqrt{2} i) / 27$,

$$
f=\frac{e^{4 \gamma}-1}{e^{\gamma}-1}, \quad g=\frac{e^{-4 \gamma}-1}{e^{-\gamma}-1}
$$

Since

$$
\begin{aligned}
& f-a=\left(e^{\gamma}-\frac{-1+\sqrt{2} i}{3}\right)^{2}\left(e^{\gamma}-\frac{-1-2 \sqrt{2} i}{3}\right) \\
& g-a=\left(e^{-\gamma}-\frac{-1+\sqrt{2} i}{3}\right)^{2}\left(e^{-\gamma}-\frac{-1-2 \sqrt{2} i}{3}\right)
\end{aligned}
$$

we have $\bar{N}_{(2}(r, 1 /(f-a))=1 / 3 T(r, f)+S(r, f)$. 
The conditions in the following theorem are stronger slightly than those in Theorem 1, but the conclusion is more clear.

THEOREM 2. Let $f$ and $g$ be non-constant meromorphic functions sharing $0,1, \infty C M$. Suppose additionally that $f$ is not a Möbius transformation of $g$ and that there exists an $a \neq 0,1, \infty$ such that

$$
\bar{N}_{1)}\left(r, \frac{1}{f-a}\right)=S(r, f),
$$

then $f$ and $g$ assume one of the following forms:

(i) $f=\frac{e^{3 \gamma}-1}{e^{\gamma}-1}, g=\frac{e^{-3 \gamma}-1}{e^{-\gamma}-1}$, with $a=\frac{3}{4}$;

(ii) $f=\frac{e^{\gamma}-1}{e^{3 \gamma}-1}, g=\frac{e^{-\gamma}-1}{e^{-3 \gamma}-1}$, with $a=\frac{4}{3}$;

(iii) $f=\frac{e^{3 \gamma}-1}{\lambda e^{2 \gamma}-1}, g=\frac{e^{-3 \gamma}-1}{\frac{1}{\lambda} e^{-2 \gamma}-1}$, with $a=-3$ and $\lambda^{3}=1$;

(iv) $f=\frac{e^{2 \gamma}-1}{\lambda e^{3 \gamma}-1}, g=\frac{e^{-2 \gamma}-1}{\frac{1}{\lambda} e^{-3 \gamma}-1}$, with $a=-\frac{1}{3}$ and $\lambda^{2}=1$;

(v) $f=\frac{e^{\gamma}-1}{\lambda e^{2 \gamma}-1}, g=\frac{e^{-\gamma}-1}{\frac{1}{\lambda} e^{-2 \gamma}-1}$, with $\lambda \neq 1$ and $4 a(1-a) \lambda=1$;

(vi) $f=\frac{e^{2 \gamma}-1}{\lambda e^{\gamma}-1}, g=\frac{e^{-2 \gamma}-1}{\frac{1}{\lambda} e^{-\gamma}-1}$, with $\lambda^{2} \neq 1$ and $a^{2} \lambda^{2}=4(a-1)$;

(vii) $f=\frac{e^{\gamma}-1}{e^{-2 \gamma}-1}, g=\frac{e^{-\gamma}-1}{e^{2 \gamma}-1}$, with $a=4$;

(viii) $f=\frac{e^{2 \gamma}-1}{e^{-\gamma}-1}, g=\frac{e^{-2 \gamma}-1}{e^{\gamma}-1}$, with $a=\frac{1}{4}$;

(ix) $f=\frac{e^{\gamma}-1}{\lambda e^{-\gamma}-1}, g=\frac{e^{-\gamma}-1}{\frac{1}{\lambda} e^{\gamma}-1}$, with $\lambda \neq \frac{1-a}{2}$ and $(1-a)^{2}+4 a \lambda=0$,

where $\gamma$ is a non-constant entire function.

Proof. Since $\bar{N}_{1)} r,(1 /(f-a))=S(r, f)$, by Lemma 3 , we have $T(r, f) \leq$ $2 \bar{N}_{(2}(r, 1 /(f-a))+S(r, f)$. Hence by Theorem $1, f$ and $g$ assume the forms in (4) and $a, s, t, \theta$ satisfy the equality (5). Since $s$ and $t$ are mutually prime, there exist two non-zero integers $p$ and $q$ such that $p s+q t=1$. From this and the proof of Theorem 1, we get

$$
e^{\gamma}=\lambda^{p} e^{q \alpha} e^{-p \beta}
$$

At point $z_{0}$, we have $e^{\alpha\left(z_{0}\right)}=(1-a) /(1-\theta)$ and $e^{\beta\left(z_{0}\right)}=(1-a) \theta / a(1-\theta)$, and thus

$$
e^{\gamma\left(z_{0}\right)}=\lambda^{p} \frac{a^{p}(1-a)^{q-p}}{\theta^{p}(1-\theta)^{q-p}}:=u .
$$

Now we discuss three cases below. 
CASE 1. $t>-s>0$.

If $t=2$, then $s=-1$, and thus $p=1, q=1$ and $\theta=2$. From (5), we get $a^{2} \lambda^{2}=4(a-1) . \quad a \neq \theta=2$ and $a^{2} \lambda^{2}=4(a-1)$ imply $\lambda^{2} \neq 1$. Hence $f$ and $g$ assume the form (vi) in Theorem 2 .

Now we suppose that $t \geq 3$. Let

$$
\varphi:=\frac{\left(e^{\gamma}-\lambda^{p}\right)\left(e^{\gamma}-\mu\right)^{2}}{e^{\alpha}-a e^{\beta}+a-1} .
$$

Since $\bar{N}_{1)}(r, 1 /(f-a))=S(r, f)$, from (11), (23) and (24), we see that $N(r, \varphi)=S(r, f)$. Since $e^{\alpha}-a e^{\beta}+a-1$ is a polynomial in $e^{\gamma}$ with degree $t \geq 3$ and non-zero constant term $a-1$, it is quite easy to see that $m(r, \varphi)=S(r, f)$. Hence we have $T(r, \varphi)=S(r, f)$. Formula (25) can be rewritten as

$$
\varphi\left(e^{t \gamma}-a \lambda e^{-s \gamma}+a-1\right)=e^{3 \gamma}-\left(\lambda^{p}+2 \mu\right) e^{2 \gamma}+\left(\mu^{2}+2 \mu \lambda^{p}\right) e^{\gamma}-\mu^{2} \lambda^{p} .
$$

From this and Lemma 2, we get $t=3$ and

$$
\varphi \equiv 1, \quad \varphi(a-1) \equiv-\mu^{2} \lambda^{p},
$$

and thus

$$
-a \lambda e^{-s \gamma}=-\left(\lambda^{p}+2 \mu\right) e^{2 \gamma}+\left(\mu^{2}+2 \mu \lambda^{p}\right) e^{\gamma} .
$$

By Lemma 2 again, we see that $-s=2$ or $-s=1$.

If $-s=2$, then from (27), we have

$$
a \lambda=\lambda^{p}+2 \mu, \quad u+2 \lambda^{p}=0 .
$$

Since $t=3$ and $-s=2$, by the definition of $p, q$, we have $p=q=1$. Hence from (26) and the above formulas, we get

$$
\lambda^{3}=1, \quad a=-3, \quad \mu=-2 \lambda .
$$

Therefore

$$
f=\frac{e^{3 \gamma}-1}{\lambda e^{2 \gamma}-1}, \quad g=\frac{e^{-3 \gamma}-1}{\frac{1}{\lambda} e^{-2 \gamma}-1},
$$

which assume the form (iii) in Theorem 2 .

If $-s=1$, then from (27) and by Lemma 2, we have

$$
\lambda^{p}+2 \mu=0, \quad-a \lambda=\mu^{2}+2 \mu \lambda^{p} .
$$

By the definition of $p, q$, we have $p=2$ and $q=1$. From (26) and the above formulas, we get

$$
\mu=-\frac{1}{2} \lambda^{2}, \quad a=\frac{3}{4} \lambda^{3}, \quad \frac{1}{4} \lambda^{6}+\frac{3}{4} \lambda^{3}=1
$$

Hence

$$
f-a=\frac{e^{3 \gamma}-\frac{3}{4} \lambda^{4} e^{\gamma}+\frac{3}{4} \lambda^{3}-1}{\lambda e^{\gamma}-1}=\frac{e^{3 \gamma}-\frac{3}{4} \lambda^{4} e^{\gamma}-\frac{1}{4} \lambda^{6}}{\lambda e^{\gamma}-1}=\frac{\left(e^{\gamma}-\lambda^{2}\right)\left(e^{\gamma}+\frac{1}{2} \lambda^{2}\right)^{2}}{\lambda e^{\gamma}-1} .
$$


Since $\bar{N}_{1)}(r, 1 /(f-a))=S(r, f)$, the above formulas lead to $\lambda^{3}=1$. Hence $a=3 / 4$ and $f=\left(e^{3 \gamma}-1\right) /\left(\lambda e^{\gamma}-1\right)$. Select an entire function $\gamma_{1}$ such that $e^{\gamma_{1}}=\lambda e^{\gamma}$, then

$$
f=\frac{e^{3 \gamma_{1}}-1}{e^{\gamma 1}-1}, \quad g=\frac{e^{-3 \gamma_{1}}-1}{e^{-\gamma_{1}}-1}, \quad a=\frac{3}{4}
$$

which assume the form (i) in Theorem 2 .

CASE 2. $-s>t>0$.

If $-s=2$, then $t=1$, and thus from (4), we have

$$
f-a=\frac{e^{\gamma}-a \lambda e^{2 \gamma}+a-1}{\lambda e^{2 \gamma}-1} .
$$

Since $\bar{N}_{1)}(r, 1 /(f-a))=S(r, f)$, from the above formula, we get

$$
1+4 a \lambda(a-1)=0
$$

and

$$
f-a=\frac{-a \lambda\left(e^{\gamma}+\frac{1}{2 a \lambda}\right)^{2}}{\lambda e^{2 \gamma}-1} .
$$

If $\lambda=1$, then $a=1 / 2$. Therefore $f-a=-\left(e^{\gamma}+1\right) / 2\left(e^{\gamma}-1\right)$, which contradicts $T(r, f) \leq 2 \bar{N}_{(2}(r, 1 /(f-a))+S(r, f)$. Hence $\lambda \neq 1$ and $f, g$ assume the form (v) in Theorem 2.

Now we consider the subcase $-s \geq 3$. Similar to Case 1 , we can prove the function $\varphi$ in $(25)$ still satisfies $T(r, \varphi)=S(r, f)$. By Lemma 2, we can get $-s=3$, and

$$
-a \lambda \varphi=1, \quad \varphi(a-1)=-\mu^{2} \lambda^{p}
$$

and thus

$$
\varphi e^{t \gamma}=-\left(\lambda^{p}+2 \mu\right) e^{2 \gamma}+\left(\mu^{2}+2 \mu \lambda^{p}\right) e^{\gamma} .
$$

If $t=2$, then from the above formula and by Lemma 2, we have

$$
\varphi=-\left(\lambda^{p}+2 \mu\right), \quad \mu^{2}+2 \mu \lambda^{p}=0 .
$$

Since $s=-3, t=2$, by the definition of $p, q$, we see that $p=q=-1$. Therefore, from the above equalities and (28), we get $a=-1 / 3$, and $\lambda^{2}=1$. Hence $f$ and $g$ assume the form (iv) in Theorem 2 .

If $t=1$, then $p=-1, q=-2$. From (29), we have

$$
\varphi=\mu^{2}+2 \mu \lambda^{p}, \quad \lambda^{p}+2 \mu=0 .
$$

This and (28) imply that

$$
a=\frac{4}{3} \lambda, \quad \mu=-\frac{1}{2 \lambda}, \quad \varphi=-\frac{3}{4 \lambda^{2}}, \quad 4 \lambda^{2}-3 \lambda-1=0 .
$$


Then we have

$$
f-a=-\frac{4}{3} \lambda \frac{\left(e^{\gamma}-\frac{1}{\lambda}\right)\left(e^{\gamma}+\frac{1}{2 \lambda}\right)^{2}}{e^{3 \gamma}-\frac{1}{\lambda}} .
$$

Since $\bar{N}_{1)}(r, 1 /(f-a))=S(r, f)$, we can get $\lambda=1$, and thus $a=4 / 3$. Hence $f$ and $g$ assume the form (ii) in Theorem 2 .

CASE 3. $t>0>-s$.

In this case, we express $f-a$ as

$$
f-a=-\frac{e^{(s+t) \gamma}+(a-1) e^{s \gamma}-\lambda a}{e^{s \gamma}-\lambda} .
$$

If $s+t=2$, then $s=t=1$, and

$$
f-a=-\frac{e^{2 \gamma}+(a-1) e^{\gamma}-\lambda a}{e^{\gamma}-\lambda}
$$

Since $\bar{N}_{1)}(r, 1 /(f-a))=S(r, f)$, the numerator in the above formula is a complete square form. Therefore

$$
(1-a)^{2}+4 a \lambda=0
$$

And then

$$
f-a=-\frac{\left(e^{\gamma}+\frac{a-1}{2}\right)^{2}}{e^{\gamma}-\lambda}, \quad \lambda \neq \frac{1-a}{2} .
$$

Hence $f$ and $g$ assume the form (ix) in Theorem 2 .

For the subcase $s+t \geq 3$, let

$$
\psi:=\frac{\left(e^{\gamma}-\lambda^{p}\right)\left(e^{\gamma}-\mu\right)^{2}}{e^{(s+t) \gamma}+(a-1) e^{s \gamma}-\lambda a} .
$$

Similar to Case 1, we can prove that $T(r, \psi)=S(r, f)$. And then it is not difficult to prove that $f$ and $g$ assume the forms (vii) and (viii) in Theorem 2. The proof of Theorem 2 is then completed.

THEOREM 3. Let $f$ and $g$ be non-constant meromorphic functions and $S_{1}=\left\{a_{1}, a_{2}\right\}, \quad S_{2}=\left\{b_{1}, b_{2}\right\}, \quad S_{3}=\{\infty\}$ satisfying $a_{1} \neq a_{2}, \quad b_{1} \neq b_{2}, \quad a_{1}+a_{2}=$ $b_{1}+b_{2}=c, a_{1} a_{2} \neq b_{2} b_{2}$. Let $d=\left(1 /\left(b_{1} b_{2}-a_{1} a_{2}\right)\right)\left(\left(a_{1}-a_{2}\right) / 2\right)^{2}$. If $E_{f}\left(S_{j}\right)=$ $E_{g}\left(S_{j}\right)(j=1,2,3)$, then $f$ and $g$ assume one of the following relations:

(i) $f \equiv g$;

(ii) $f+g \equiv c$; 
(iii) $f=\frac{c}{2} \pm\left(b_{1}-b_{2}\right)\left(e^{\gamma}+\frac{1}{2}\right), g=\frac{c}{2} \pm\left(b_{1}-b_{2}\right)\left(e^{-\gamma}+\frac{1}{2}\right)$ with $d=\frac{3}{4}$;

(iv) $f=\frac{c}{2} \pm\left(a_{1}-a_{2}\right)\left(e^{\gamma}+\frac{1}{2}\right), g=\frac{c}{2} \pm\left(a_{1}-a_{2}\right)\left(e^{-\gamma}+\frac{1}{2}\right)$ with $d=\frac{1}{4}$;

(v) $f=\frac{c}{2} \pm \frac{a_{1}-a_{2}}{2} e^{\gamma}$ and $g=\frac{c}{2} \pm \frac{a_{1}-a_{2}}{2} e^{-\gamma}$ with $d=\frac{1}{2}$,

where $\gamma$ is a non-constant entire function.

Proof. Since $E_{f}\left(S_{j}\right)=E_{g}\left(S_{j}\right)(j=1,2,3)$, there exist two entire functions $\alpha$ and $\beta$ such that

$$
\frac{\left(f-a_{1}\right)\left(f-a_{2}\right)}{\left(g-a_{1}\right)\left(g-a_{2}\right)}=e^{\alpha}, \quad \frac{\left(f-b_{1}\right)\left(f-b_{2}\right)}{\left(g-b_{1}\right)\left(g-b_{2}\right)}=e^{\beta},
$$

from which we have

$$
\frac{(f-g)\left(f+g-a_{1}-a_{2}\right)}{\left(g-a_{1}\right)\left(g-a_{2}\right)}=e^{\alpha}-1,
$$

and

$$
\frac{(f-g)\left(f+g-b_{1}-b_{2}\right)}{\left(g-b_{1}\right)\left(g-b_{2}\right)}=e^{\beta}-1 .
$$

Since $a_{1}+a_{2}=b_{1}+b_{2}=c$, we see that $f \equiv g$ or $f+g \equiv c$ as long as one of $e^{\alpha} \equiv 1, e^{\beta} \equiv 1$ and $e^{\alpha} \equiv e^{\beta}$ hold. In the following, we suppose that $e^{\alpha} \not \equiv 1$, $e^{\beta} \not \equiv 1$ and $e^{\alpha-\beta} \not \equiv 1$.

Since $f$ and $g$ share $S_{i}(i=1,2,3) \mathrm{CM}$, by the second fundamental theorem, we have

$$
\begin{aligned}
T(r, f) & <N(r, f)+N\left(r, \frac{1}{f-a_{1}}\right)+N\left(r, \frac{1}{f-a_{2}}\right)+S(r, f) \\
& =N(r, g)+N\left(r, \frac{1}{g-a_{1}}\right)+N\left(r, \frac{1}{g-a_{2}}\right)+S(r, f) \\
& \leq 3 T(r, g)+S(r, f) .
\end{aligned}
$$

Similarly, we have $T(r, g)<3 T(r, f)+S(r, g)$. Hence $S(r, f)=S(r, g)$. Write $S(r):=S(r, f)=S(r, g)$. From (30), we can get

$$
T\left(r, e^{\alpha}\right) \leq 8 T(r, f)+S(r), \quad T\left(r, e^{\beta}\right) \leq 8 T(r, f)+S(r) .
$$

Therefore

$$
T(r, \alpha) \leq S(r), \quad T(r, \beta) \leq S(r) .
$$

From (31) and (32), we can easily get 


$$
\frac{a_{1} a_{2}-b_{1} b_{2}}{\left(g-b_{1}\right)\left(g-b_{2}\right)}=\frac{e^{\beta}-e^{\alpha}}{e^{\alpha}-1} \text {. }
$$

Therefore the poles of $g$ are multiple zeros of $e^{\beta}-e^{\alpha}$, and thus the zeros of $\beta^{\prime}-\alpha^{\prime}$. If $\beta^{\prime}-\alpha^{\prime} \equiv 0$, then $e^{\alpha-\beta}$ is a constant different from 1 . Hence $g$ have no poles in this case. If $\beta^{\prime}-\alpha^{\prime} \not \equiv 0$, then

$$
\begin{aligned}
N(r, g) & \leq N\left(r, \frac{1}{\alpha^{\prime}-\beta^{\prime}}\right) \leq T\left(r, \alpha^{\prime}-\beta^{\prime}\right)+O(1) \\
& =S(r) .
\end{aligned}
$$

In both cases, we have

$$
N(r, g)=S(r)
$$

Similarly, we have

$$
N(r, f)=S(r)
$$

Let

$$
F:=\frac{\left(f-a_{1}\right)\left(f-a_{2}\right)}{a_{1} a_{2}-b_{1} b_{2}}, \quad G:=\frac{\left(g-a_{1}\right)\left(g-a_{2}\right)}{a_{1} a_{2}-b_{1} b_{2}}
$$

Since

$$
F-1=\frac{\left(f-b_{1}\right)\left(f-b_{2}\right)}{a_{1} a_{2}-b_{1} b_{2}}, \quad G-1=\frac{\left(g-b_{1}\right)\left(g-b_{2}\right)}{a_{1} a_{2}-b_{1} b_{2}},
$$

we see that $F$ and $G$ share $0,1, \infty \mathrm{CM}$. Let

$$
d:=\left(\frac{a_{1}-a_{2}}{2}\right)^{2} \frac{1}{b_{1} b_{2}-a_{1} a_{2}} .
$$

It is quite easy to verify that $d \neq 0,1, \infty$. From the two equalities in (36), we deduce that

$$
\begin{aligned}
& F-d=\frac{1}{a_{1} a_{2}-b_{1} b_{2}}\left(f-\frac{c}{2}\right)^{2}, \\
& G-d=\frac{1}{a_{1} a_{2}-b_{1} b_{2}}\left(g-\frac{c}{2}\right)^{2} .
\end{aligned}
$$

Hence $N_{1)}(r, 1 /(F-d))=N_{1)}(r, 1 /(G-d))=0$. From (35), (37) and the second fundamental theorem, we get

$$
\bar{N}\left(r, \frac{1}{F-1}\right) \geq T(r, f)+S(r) .
$$


Hence $\bar{N}(r, 1 /(F-1)) \neq S(r, F)$. Similarly, $\bar{N}(r, 1 / F) \neq S(r, F)$. By Theorem 2 , one of the following cases holds:

(A) $F=e^{2 \gamma}+e^{\gamma}+1, G=e^{-2 \gamma}+e^{-\gamma}+1, d=\frac{3}{4}$.

(B) $F=-e^{\gamma}\left(e^{\gamma}+1\right), G=-e^{-\gamma}\left(e^{-\gamma}+1\right), d=\frac{1}{4}$.

(C) $F$ is a Möbius transformation of $G$,

where $\gamma$ is a non-constant entire function.

In Case $\mathrm{A}$, we have $F-d=\left(e^{\gamma}+1 / 2\right)^{2}, G-d=\left(e^{-\gamma}+1 / 2\right)^{2}$. Hence

$$
\left(f-\frac{c}{2}\right)^{2}=\left(a_{1} a_{2}-b_{1} b_{2}\right)\left(e^{\gamma}+\frac{1}{2}\right)^{2}, \quad\left(g-\frac{c}{2}\right)^{2}=\left(a_{1} a_{2}-b_{1} b_{2}\right)\left(e^{-\gamma}+\frac{1}{2}\right)^{2} \text {. }
$$

Since $d=3 / 4$, we have $\left(a_{1}-a_{2}\right)^{2}=3\left(b_{1} b_{2}-a_{1} a_{2}\right)$. Combining this and $a_{1}+a_{2}=b_{1}+b_{2}$, we get $a_{1} a_{2}-b_{1} b_{2}=\left(b_{1}-b_{2}\right)^{2}$. Hence

$$
f=\frac{c}{2} \pm\left(b_{1}-b_{2}\right)\left(e^{\gamma}+\frac{1}{2}\right), \quad g=\frac{c}{2} \pm\left(b_{1}-b_{2}\right)\left(e^{-\gamma}+\frac{1}{2}\right) \text {. }
$$

Similarly, in Case B, we have

$$
F-d=-\left(e^{\gamma}+\frac{1}{2}\right)^{2}, \quad G-d=-\left(e^{-\gamma}+\frac{1}{2}\right)^{2}
$$

and

$$
d=\frac{1}{4}, \quad a_{1} a_{2}-b_{1} b_{2}=-\left(a_{1}-a_{2}\right)^{2} .
$$

Hence

$$
f=\frac{c}{2} \pm\left(a_{1}-a_{2}\right)\left(e^{\gamma}+\frac{1}{2}\right), \quad g=\frac{c}{2} \pm\left(a_{1}-a_{2}\right)\left(e^{-\gamma}+\frac{1}{2}\right) .
$$

Now we discuss Case C. Since $F$ is a Möbius transformation of $G$, and $F$ and $G$ share $0,1, \infty \mathrm{CM}$, furthermore $N(r, F)=S(r, F), N(r, 1 / F) \neq S(r, F)$ and $N(r, 1 /(F-1)) \neq S(r, F)$, we can see that there exists a $d_{1} \neq 0,1, \infty$ such that $d_{1}, \infty$ are exceptional values of $F$. Since $N_{1)}(r, 1 /(F-d))=0$, by the second fundamental theorem, we can deduce that $d_{1}=d$. Hence $\infty, c / 2$ are two exceptional values of $f$. Similarly, $\infty, c / 2$ are two exceptional values of $g$. We have assumed that $e^{\alpha} \not \equiv 1$, which is equivalent to $F \not \equiv G$. Hence we have

$$
(F-d)(G-d)=d^{2} \quad \text { and } \quad d=\frac{1}{2},
$$

which imply that

$$
\left(f-\frac{c}{2}\right)\left(g-\frac{c}{2}\right)= \pm\left(\frac{a_{1}-a_{2}}{2}\right)^{2}
$$


Hence

$$
f=\frac{c}{2} \pm \frac{a_{1}-a_{2}}{2} e^{\gamma}, \quad g=\frac{c}{2} \pm \frac{a_{1}-a_{2}}{2} e^{-\gamma}
$$

which completes the proof of Theorem 3.

Acknowledgement. The author wants to thank the referee for his/her thorough reviewing with useful suggestions and comments made to the paper.

\title{
REFERENCES
}

[1] W BergWeILER, On meromorphic functions that share three values and on the exceptional set in Wiman-Valiron theory, Koda1 Math. J., 13 (1990), pp. 1-9.

[2] G. Brosch, Eindeutigkeitssätze für meromorphe Funktionen, Thesis, Technical University of Aachen, 1989.

[3] F. Gross AND C. C. YANG, Meromorphic functions coverıng certain finite sets at the same points, Illinois J. Math., 26 (1982), pp. 432-441.

[4] E. MUEs, Shared value problems for meromorphic functions, Value Distribution Theory And Complex Differential Equations, Joensuu, 1994, Univ. Joensuu, 1995, pp. 17-43.

[5] R. Nevanlinna, Einıge Eindentigkeitssätze in der Theorıe der Meromorphen Funktionen, Acta Math., 48 (1926), pp. 367-391.

[6] P. LI AND C. C. YANG, On the characteristics of meromorphic functions that share three values CM, J. Math. Anal. Appl., 220 (1998), pp. 132-145.

[7] U. StrahmanN, Eindeutigkeitssätze für meromorphe Funktıonen, die nebst ihren Ableitungen Werte teilen, Thesis, University of Hannover, 1993.

[ 8] H. UEDA, Unicity theorems for meromorphic or entire functions II, Koda1 Math. J., 6 (1983), pp. 26-36.

[9] S.-Z. YE, Uniqueness of meromorphic functions that share three values, Koda1 Math. J., 15 (1992), pp. 236-243.

[10] H. X. YI, On the meromorphic function that cover three finite sets at same points, J. Shandong Unıv. Nat. Sci. Ed., 22 (1987), pp. 41-53 (in Chınese).

[11] H. X. YI, Meromorphic functions that share two or three values, Koda1 Math. J., 13 (1990), pp. 363-372.

[12] H. X. YI AND C. C. YANG, Uniqueness Theory of Meromorphic Functions, Science Press, China, 1995 (in Chinese).

\author{
DePartment of Mathematics \\ The University of Science and Technology of China \\ Hefei, ANHui, 230026 \\ P. R. CHINA \\ email: pli@math.ustc.edu.cn
}

